\title{
Guardando al futuro. La questione dei 4 miliardi
}

\author{
Massimo Livi-Bacci
}

\author{
Università di Firenze, Italia
}

\begin{abstract}
Sommario
La popolazione interagisce con le costrizioni esterne, quali lo spazio, il suolo, l'acqua, l'aria, le risorse non rinnovabili e l'energia. L'umanità, nella sua storia, ha trovato queste risorse in quasi illimitata quantità. Ma le cose sono cambiate rapidamente, e alcune risorse naturali - particolarmente il suolo, l'acqua e l'aria - sono in tensione a causa del rapido aumento della popolazione. Durante l'eccezionale XX secolo, la popolazione del mondo si è quadruplicata, durante l'attuale secolo andrà vicina al raddoppio. La forza principale del riscaldamento globale sia di natura antropogenica, legata all'aumento delle attività umane, l'altra metà essendo imputabile alla crescita del PIL pro-capite. D'altra parte, una popolazione in espansione richiede più terra e spazio in un mondo finito. Man mano che la popolazione cresce, lo spazio disponibile diminuisce e l'antropizzazione della terra aumenta. Il documento discute le implicazioni dell'interazione tra popolazione, riscaldamento globale e antropizzazione della terra.
\end{abstract}

Parola chiave: Proiezioni della popolazione, riscaldamento globale, antropizzazione.

Abstract

Looking at the future. The question of 4 billion

Population interacts with the external constraints such as space, land, water, air, non-renewable resources and energy. Humankind, throughout its history on this planet, has found these resources in almost unlimited supply. But things have changed rapidly in recent times and some natural resources - particularly air, land and water - are under stress because of rapid population growth. During an exceptional twentieth century, the world population increased fourfold; during the current century it will fall short of doubling. Global warming is primarily "anthropogenic", or due to the increase in the volume of human activities, that are a function of the increase of the number of people and of their per-capita consumption. On the other hand, an expanding population requires more land and space in a finite world. As population grows, the available space declines, and anthropization of land increases. The paper discusses the implications of the interaction between population, global warming and anthropization of land.

Key words: Population projections, global warming, anthropization. 


\section{IL PENDOLO DEI TIMORI}

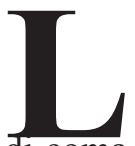

a "questione demografica", che è stata al centro del dibattito circa le sorti dell'umanità fin dal XVIII secolo, è scivolata fuori

dalle luci della ribalta e negli ultimi anni è entrata in una sorta di coma. Per secoli, il pensiero dominante ha oscillato, come un pendolo, tra una visione positiva, ed una negativa, del numero e della crescita. Nel XVII e nel XVIII secolo, il mercantilismo aveva sostenuto la necessità e l'opportunità di una popolazione numerosa ed in crescita, come fattore vantaggioso sotto il profilo politico, economico e militare. La ricchezza di una nazione era legata alla sua numerosità, ed al margine esistente tra i consumi della popolazione, prevalentemente rurale, ed il valore del prodotto. Un margine che tramite l'imposizione di tributi accresceva le risorse dello stato, e quindi la sua potenza. "Una popolazione numerosa e crescente era il sintomo più importante di ricchezza; anzi, era la causa più importante della ricchezza, essa stessa era ricchezza, il più grande patrimonio che una nazione potesse avere" (Schumpeter, 1959). Ma già durante il XVIII secolo le convinzioni dei mercantilisti iniziarono a sgretolarsi, e si fece di nuovo strada l'antica idea che la crescita della popolazione fosse determinata da1la disponibilità di sussistenze e che non fosse, in sé, un fatto positivo. E' in questo contesto che scrive Malthus, dando una spinta decisiva al pendolo verso il polo negativo: la mancanza di freni preventivi alla crescita determina una pressione sulle risorse e pone in azione i freni repressivi negativi atti a riportare la popolazione su livelli più bassi. Per larga parte del XIX secolo la posizione di Malthus, con articolazioni e approfondimenti, permea gli scritti in materia di economia e società. Il mondo è finito, la terra è finita, la crescita demografica non può essere illimitata. "Affermo che il potere (generativo) della popolazione è infinitamente più grande della capacità della terra di produrre sussistenze per gli uomini" (Malthus, 1970). La lettura di Malthus ispirò Darwin, e contribuì alla nascita della teoria evoluzionista e della selezione naturale, come conseguenza della lotta per la sopravvivenza. Si fece poi spazio l'idea che, in un mondo finito ma con spazi aperti, la specie umana, come le specie animali, abbia bisogno di spazi vitali - lebensraum - da occupare ed insediare. Un concetto elaborato da Ratzel (1897) zoologo, geografo e antropologo, che verrà poi assunto e distorto dal nazismo e da altri regimi dittatoriali. Per quasi tutto il XIX secolo, punteggiato ancora da crisi di sussistenza e carestie, dall'emigrazione 
di massa e dall'emergere di nuove povertà, la crescita della popolazione e del suo numero assunse un connotato non positivo.

Ma il pendolo era sul limite di un'altra oscillazione : "Tra 14 anni la Germania avrà un numero di coscritti doppio di quello della Francia; allora quel popolo che ci detesta ci divorerà: i tedeschi lo affermano, lo instillano e lo faranno" (Spengler, 1938) scriveva Jacques Bertillon, illustre scienziato sociale, nel 1897. La ferita della sconfitta militare di Sedan nel 1870 era ancora aperta. La natalità che era in diminuzione in Francia già dall'inizio del secolo, cominciò a declinare in altre aree dell'Europa più sviluppata. La demografia assunse una valenza politica: 1'Europa aveva esportato verso gli altri continenti milioni di migranti e le "grandi potenze" avevano costruito estesi imperi coloniali, imitate tardivamente da Germania, Italia e Giappone. Il numero e lo spazio diventarono elementi importanti nella geopolitica. Ci fu diffusa attenzione e anche preoccupazione per il diffondersi del declino della natalità. Politiche pro-nataliste vennero poste in atto in vari paesi e non solo nei paesi governati da dittature. La necessità dello "spazio vitale" diventò l'argomento pretestuoso dell'espansione nazista in Europa. Il numero si legava alla potenza militare, all'influenza internazionale, all'espansionismo territoriale.

Nel famoso "Discorso dell'Ascensione", del Maggio del 1927, Mussolini riassunse in modo incisivo i timori e le opinioni che serpeggiavano nel mondo occidentale. Conviene citarlo.

Qualche inintelligente dice: "Siamo in troppi". Gli intelligenti rispondono: "Siamo in pochi". Affermo che, dato non fondamentale ma pregiudiziale della potenza politica, e quindi economica e morale delle Nazioni, è la loro potenza demografica. ${ }^{1}$

Negli anni '20 e '30, le perdite umane della prima guerra mondiale, il declino della natalità nei paesi più ricchi, la prospettiva - seppur lontana - di un indebolimento demografico, apparvero indizi o prove che il crepuscolo della civiltà occidentale stesse avverandosi. La questione dei rapporti tra dimensioni e velocità di crescita della popolazione, e la potenza delle nazioni, era antica, ed aveva assunto nel tempo le molteplici

\footnotetext{
${ }^{1}$ Benito Mussolini, Il discorso dell'Ascensione, Libreria Littorio, 1927. Il discorso continuava così: "Parliamoci chiaro: che cosa sono 40 milioni d'Italiani di fronte a 90 milioni di Tedeschi e a 200 milioni di Slavi? Volgiamoci a Occidente: che cosa sono 40 milioni di Italiani di fronte a 40 milioni di Francesi, più i 90 milioni di abitanti delle Colonie, o di fronte ai 46 milioni di Inglesi, più i 450 milioni che stanno nelle Colonie? Signori, l'Italia, per contare qualche cosa, deve affacciarsi sulla soglia della seconda metà di questo secolo con una popolazione non inferiore ai 60 milioni di abitanti"
} 
forme sopra ricordate, prima che Mussolini la riprendesse in modo diretto e brutale.

Nell'ultimo dopoguerra, il pendolo compie due altre oscillazioni. In Occidente c'è una forte ripresa della crescita demografica, alimentata dal baby boom dagli anni '40 agli anni '60; c'è la definitiva scoperta statistica di un mondo povero con un alto ritmo di crescita mentre la decolonizzazione sottrae gran parte di quel mondo al controllo politico diretto dell'occidente. Si accresce il timore circa i pericoli del deterioramento dell' ambiente ed il possibile esaurirsi di risorse non rinnovabili. Crescita e numero assumono valori negativi. Tra la fine degli anni ' 60 e l'inizio degli '70, l'opinione pubblica - particolarmente nel mondo anglosassone- è sommersa da allarmi catastrofisti: nel 1968 Paul Ehrlich, un biologo, scrive "The Population Bomb", e basta il titolo per sintetizzarne il messaggio ("non possiamo permetterci di curare i sintomi del cancro della crescita demografica: bisogna estirpare lo stesso cancro") (Ehrlich, 1968). Nel 1972 il Club di Roma produce un rapporto sui limiti dello sviluppo, preconizzando la catastrofe industriale e demografica entro cento anni in mancanza di misure draconiane (Meadows et al., 1972). La crescita demografica evoca appellativi bellici: bomba, esplosione, boom, sfida... E' significativo che gli anni '70 si aprano con gli allarmi del movimento ZPG (Zero Population Growth) in merito all'aumento demografico degli Stati Uniti, e si chiudano con l'introduzione in Cina della politica del figlio unico, volta a frenare, e eventualmente invertire, la crescita demografica. Per il paesi più popoloso del mondo si trattò dell'aperto riconoscimento che le grandi dimensioni, e la rapida crescita, sono fattori primari di debolezza, e non di forza.

L'ultima oscillazione del pendolo è storia degli ultimi due o tre decenni: si è riaffacciato, come all'inizio del secolo scorso, il timore del declino demografico, alimentato dalla caduta della riproduttività ben al di sotto del livello di rimpiazzo delle generazioni in buona parte del mondo sviluppato, e dal diffondersi di comportamenti riproduttivi analoghi in molti paesi del mondo povero. Si teme anche che questa situazione sia difficilmente reversibile considerato la scarsa efficacia e l'alto costo delle politiche sociali ed economiche volte a rianimarla, là dove si è tentato di farlo.

Questa breve rassegna stilizzata delle paure e delle speranze indotte dal cambio demografico è una semplificazione di un percorso assai complesso. Giovanni Botero, le cui idee ebbero ampia risonanza, fu un precursore di Malthus, agli albori del mercantilismo; alla metà del secolo XIX, in piena epoca neomalthusiana, l'America è sicuramente popolazionista, e uno dei padri della costituzione argentina, nel 1852, sintetizzava lo spirito 
dei tempi con la massima "gobernar es poblar" (Bautista Alberdi, 1852). Negli anni '20 e '30 del '900, molti sostenitori delle teorie eugenetiche furono fortemente neo-malthusiani; e nei due o tre decenni successivi alla Seconda Guerra Mondiale vigeva l'idea, in diversi paesi in via di sviluppo, che la crescita della popolazione fosse un fenomeno positivo perché rafforzava il mercato interno e l'indipendenza dall'economia mondiale dominata dall'occidente. Ancor oggi, il pendolo oscilla verso il timore del declino demografico in Europa e nell'oriente asiatico, contemporaneamente all'oscillazione in senso contrario di un altro pendolo, verso la paura per la debordante crescita demografica dell'Africa sub-Sahariana.

\section{LA QUESTIONE DEMOGRAFICA E LA COMUNITÀ INTERNAZIONALE}

Nell'ultimo mezzo secolo, la comunità internazionale ha alimentato e tentato di coordinare il dibattito sulla questione demografica, indirizzandolo all'elaborazione di soluzioni condivise. Tentativi di difficile esito perché la demografia tocca temi eticamente e politicamente molto sensibili come la riproduzione, il controllo delle nascite, le forme familiari, le migrazioni internazionali; non esiste inoltre consenso se, per una data società, sia meglio avere una popolazione più grande od una più piccola. E' difficile dunque trovare un consenso. Non che sia più facile trovare il consenso su altre questioni internazionali: ma sulla salute, per esempio, c'è pieno accordo che i microbi e i virus siano gli avversari da battere; o sul clima -a parte sparute minoranze che ritengono che il riscaldamento globale non sia dovuto a forze antropogeniche - tutti concordano sul fatto che l'emissione dei gas serra sia il nemico da "abbattere". Tuttavia l'azione della comunità internazionale ha portato, se non altro, alla piena conoscenza dei meccanismi del cambio demografico, delle loro determinanti, e delle opzioni per contrastare tendenze ritenute dannose. Le conferenze politiche delle Nazioni Unite del 1974 (Bucarest), del 1984 (Città del Messico) e del 1994 (Il Cairo) — quest'ultima con l'approvazione di un Plan of Action on Population and Development - furono occasioni importanti per la definizione di azioni condivise in merito ad uno sviluppo demografico troppo vigoroso e, comunque, fonte di squilibri sociali. Esaurito il ciclo delle conferenze politiche internazionali di tema demografico (anche se l'ultima, quella del Cairo, incorporava i temi dello sviluppo, intitolandosi Conference on Population and Development), le Nazioni Unite hanno affrontato la complessità dei temi dello sviluppo e della sua sostenibilità con il solenne appello dei Capi di Stato, nel 2000, al conseguimento dei Millennium Development Goals (MDG) nel quindicennio successivo 
(2000-2015). Si trattava di 8 grandi obbiettivi (Goals), articolati in 17 traguardi (Targets) quantificabili e monitorabili da 48 indicatori. Le questioni demografiche sono ancora ben presenti negli MDG, che però sembrano evaporate nel più ambizioso piano approvato nel 2015, e riferito al quindicennio 2015-2030. Nel Settembre 2015, infatti, un paio di centinaia di Capi di Stato convenuti all'Assemblea Generale delle Nazioni Unite, si sono impegnati a perseguire i cosiddetti Sustainable Development Goals (SDG), fortemente inflazionati rispetto al 2000, essendo cresciuti a 17, articolati in ben 169 traguardi, monitorabili con ben 270 indicatori, molti dei quali ancora inesistenti, o difficilmente calcolabili, o non significativi. ${ }^{2}$ E' davvero dubbio che un ventaglio così ampio di obbiettivi, senza priorità, che vanno dall'irrilevante all'evanescente, possa condensarsi in motivazioni, parole d'ordine, linee guida in grado di mobilitare coscienze, risorse e azioni. $\mathrm{Si}$ tratta purtroppo di uno zibaldone che rispecchia le procedure burocratiche delle istituzioni internazionali, nel quale hanno avuto un ruolo primario $i$ cosiddetti stakeholder, portatori di esigenze specifiche, sena che queste siano state selezionarle e ordinate secondo le priorità. Comunque sia, la comunità internazionale è adesso indaffarata nel promuovere i 17 SDG, col loro corredo di 169 bersagli da centrare nel 2030, e sembra convinta che la popolazione non sia più una minaccia per un equilibrato sviluppo. C'è una sorta di "consenso", tra i demografi, che la popolazione del mondo possa convergere verso uno stato semi-stazionario all'inizio del prossimo secolo, e questa convinzione ha dissolto le ansie circa il futuro nutrite da gran parte degli esperti di questioni demografiche nella seconda parte del secolo scorso.

\section{PROSSIMI QUATTRO MILIARDI}

Tre recenti articoli di David Lam (2017), Richard Grossman (2017) e George Martine (2017) hanno riaperto il dibattito circa il futuro della popolazione mondiale e le sue implicazioni per gli equilibri del pianeta. David Lam è dell'opinione che "L'ottimismo circa la capacità del mondo di accogliere i prossimi quattro miliardi di persone è conseguenza del successo col quale sono stati accolti i precedenti quattro miliardi." Richard Grossman respinge l'ottimismo di Lam, e si dice convinto che "l'aumento di quattro miliardi avvenuto nell'ultimo mezzo secolo ha degradato il mondo naturale dal quale dipendiamo, e che questo degrado renderà il mondo assai meno accogliente per i 4 miliardi che verranno." George Martine è molto più pessimista e ritiene che "data la tendenza al degrado causata dal

\footnotetext{
${ }^{2}$ Riferimenti a NU, MLB ecc.
} 
terzo più ricco della popolazione globale, il pianeta che conosciamo potrà benissimo essere distrutto anche senza l'aggiunta di un solo bambino". Questo è il classico esempio del dualismo ottimismo-pessimismo: i fatti non sono messi in dubbio, e gli autori accettano come plausibili i risultati delle proiezioni demografiche delle Nazioni Unite, e su questi basano le loro considerazioni.

Il cambiamento demografico è un processo. Questo non deve essere dimenticato: la popolazione interagisce con le costrizioni esterne, quali lo spazio, il suolo, l'acqua, l'aria, le risorse non rinnovabili e l'energia. L'umanità, nella sua storia, ha trovato queste risorse in quasi illimitata quantità. Ma le cose sono cambiate rapidamente, e alcune risorse naturali - particolarmente il suolo, l'acqua e l'aria - sono in tensione a causa del rapido aumento della popolazione. Durante l'eccezionale XX secolo, la popolazione del mondo si è quadruplicata (da 1.6 a 6.1 miliardi); durante l'attuale secolo andrà vicina al raddoppio (11.2 miliardi nel 2100, secondo la variante media delle proiezioni delle NU), e si avvicinerà ad una crescita zero alla fine di questo periodo (United Nations, 2017). Al momento di scrivere queste righe, il 30 per cento dell'aumento totale previsto per il XXI secolo è stato acquisito (alla fine del 2017, la popolazione ha raggiunto i 7.6 miliardi, 1.5 miliardi in più del 2000, su un incremento totale del secolo di 5,1 miliardi). Dal momento che il cambio demografico è processo complesso, la questione da discutere è se questo cambiamento sia compatibile con un equilibrato sviluppo. In breve: le tendenze correnti e quelle prevedibili minacciano la sostenibilità? La mia tesi è che ci sono diversi aspetti delle tendenze in atto che possono compromettere lo sviluppo e la sua sostenibilità, l'ordine politico, le relazioni tra paesi (Livi Bacci, 2017). Tra queste tendenze, variamente minacciose per la stabilità e la sostenibilità, si annoverano la rapidissima crescita dell'Africa, la depressione delle nascite in Europa e nell'Asia orientale, le migrazioni internazionali senza governo, la saturazione dello spazio, l'impatto ambientale antropogenico. D'altro canto, queste tendenze negative possono essere attenuate, se non rimediate, con politiche appropriate adottate dai governi con il sostegno della comunità internazionale. Delle varie minacce alla sostenibilità, ne commenterò brevemente due: le conseguenze ambientali della lotta alla arretratezza e alla povertà, e l'aumento dell'antropizzazione della terra.

\section{Popolazione e riscaldamento globale}

La comunità scientifica è oggi concorde nel ritenere che la forza principale del riscaldamento globale sia di natura antropogenica, legata all'aumento 
delle attività umane. Popolazione più numerosa, e più ricca, significa maggiori quantità di prodotto e di consumo, maggiori emissioni, accresciuto effetto serra, clima più caldo. Sappiamo anche che lo sviluppo della tecnologia può svincolare la crescita economica dai livelli insostenibili di produzione e consumo. In altre parole, con più tecnologia è possibile abbassare il contenuto di energia e di materie prime non rinnovabili per ogni unità prodotta o consumata. Questo può avvenire nelle società ricche, nelle quali la dematerializzazione del consumo è possibile (un dollaro aggiuntivo può essere speso per comprare un e-book, assistere ad un concerto, acquistare servizi personali); ma è molto più difficile che avvenga nelle società povere, nelle quali un dollaro addizionale viene speso per comprare gasolio per riscaldarsi, per cucinare o per trasporto; utensili di metallo per il lavoro, scarpe per camminare, ed altri prodotti di base per i quali la dematerializzazione è impossibile o minima (Bacci, 2015b).

Il recente passato ci mostra la rilevanza dell'azione antropogenica sule emissioni di gas serra. La scomposizione dell'aumento totale di $\mathrm{CO}_{2}$ proveniente dalla combustione di energia fossile, decennio per decennio, tra il 1970 e il 2010, fornisce un risultato assai interessante (International Panel on Climate Change, 2014). La componente "crescita della popolazione" sull'aumento delle emissioni è responsabile all'incirca della metà di tale aumento, l'altra metà essendo imputabile alla crescita del PIL pro-capite. Questa equivalenza vale per gli anni '70 e '90; la crescita della popolazione contribuisce invece per quasi i due terzi dell'aumento netto delle emissioni negli anni '80, e all'incirca un terzo nel primo decennio di questo secolo. L'aumento delle emissioni è al netto dell'effetto riduttivo delle emissioni dovuto al progresso tecnologico (misurato dall'intensità energetica contenuta in ogni unità di prodotto), che grosso modo controbilancia l'effetto popolazione. E' evidente che la crescita della popolazione mondiale, pur in rallentamento, continuerà ad essere responsabile nei prossimi decenni di una quota dell'aumento delle emissioni di un equivalente ordine di grandezza. Più lenta la crescita della popolazione, più lenta la crescita delle emissioni.

Si consideri il continente sub-Sahariano, nel quale il numero medio di figli per donna è oggi pari a 5 e che, secondo le proiezioni delle Nazioni Unite (variante media), scenderà a 3.1 nel 2050; se, con politiche adeguate potesse essere ridotto a 2.6 (come nella variante bassa delle proiezioni), la popolazione nel 2050 sarebbe di 1959 miliardi invece di 2 168, frenando significativamente la crescita delle emissioni. La riduzione della velocità della crescita demografica avrebbe un effetto analogo a quello del progresso tecnologico. 
Le dinamiche demografiche, quelle economiche e quelle tecnologiche, interagiscono tra loro in maniera variabile e debolmente prevedibile dai modelli. Ricordiamo ciò che è peraltro ben noto: i paesi ricchi contribuiscono più che proporzionalmente della loro popolazione al depauperamento delle riserve di risorse non rinnovabili. ${ }^{3}$ Per quanto riguarda il futuro, occorre considerare che nei paesi ricchi i processi di sostituzione, di riciclaggio e i mutamenti nei modelli di consumo potrebbero determinare una diminuzione nel contenuto di energia e materie prime in ciascun euro addizionale di produzione. Inoltre, le popolazioni di questi paesi cresceranno lentamente o rimarranno stazionarie nei prossimi decenni. Le prospettive, pertanto, di una stabilizzazione, o addirittura di un declino dei consumi di risorse di base non sono infondate. Ma le prospettive per i paesi poveri, il cui reddito è una piccola frazione di quello dei paesi ricchi, sono diverse. Nei prossimi decenni la loro velocità di sviluppo dovrà superare quella delle economie ricche affinché il rapporto (se non la differenza assoluta) tra il benessere dei due mondi possa ridursi. Nel corso della prossima generazione il prodotto pro capite di queste economie dovrà accrescersi di due, tre o più volte e questo implicherà più ferro per utensili, più fibre per vestirsi, più legname per costruire, più spazio per vivere e più energia per tutte queste attività. Poiché il livello di vita delle popolazioni povere è molto basso, questo flusso addizionale di beni per persona dovrà essere ottenuto con alti input di energia, materie prime, spazio per ogni euro di prodotto. E queste popolazioni, naturalmente, aspirano a più cibo, utensili, vestiario, abitazioni e combustibili. Considerando che in capo a un paio di generazioni esse saranno aumentate di tre miliardi, e che il flusso di beni per persona si sarà moltiplicato molte volte, è facile capire che questa pur indispensabile crescita non possa essere sostenuta a lungo.

La logica della cosiddetta "curva ambientale di Kuznets" (che si ispira ad alcune idee dell'economista Kuznets, espresse negli anni '50) aiuta ad interpretare le tendenze in corso (Livi Bacci, 2015a: 83). La curva prevede che al crescere del reddito si accresca il contenuto (materiale, energetico) di ogni unità di prodotto, ma a tassi decrescenti, fino a raggiungere un punto di svolta, oltre il quale ogni ulteriore unità di reddito avrà un contenuto decrescente di risorse. La curva assume, perciò, la forma di una campana o, meglio, di una scodella rovesciata. Alla lunga, anche i paesi poveri —allo-

\footnotetext{
${ }^{3}$ United Nations Environment Programme-UNEP, Decoupling natural resource use and environmental impacts from economic growth, A Report of the Working Group on Decoupling to the International Resource Panel. 2011. Secondo lo studio, nei paesi "poveri", nel 2000, si "estraevano" (e presumibilmente si consumavano) 18 tonnellate di materiali (biomasse, minerali, inerti da costruzione, materiali energetici) per persona nei paesi ricchi, ed appena sei nei paesi poveri.
} 
ra non più poveri- potranno percorrere il ramo discendente della curva, così come comincia ad avvenire (almeno per taluni consumi) nei paesi ricchi. Ma perché questo processo conduca all'arresto della crescita dei consumi di risorse di base, occorrerà che trascorrano varie generazioni e che la popolazione si avvii alla stazionarietà. Molto tempo dovrà passare perché questo avvenga.

\section{LA Terra è FINITA}

Una popolazione in crescita necessita più spazio e più terra, su un pianeta che è finito. Al crescere della popolazione, lo spazio disponibile diminuisce e l'antropizzazione della superficie terrestre aumenta. Oggi più della metà delle terre emerse è interessata, direttamente o indirettamente, dal processo di antropizzazione: il 13 per cento di queste per la coltivazione; il 26 per cento per pascoli, 1.8 per cento per foreste con fini produttivi, il quattro per cento per infrastrutture ed attività economiche; il tre per cento per insediamenti urbani. Nel complesso, il 54 per cento delle terre emerse è direttamente o indirettamente toccato dalle attività umane. Del rimanente 46 per cento delle terre emerse in stato (relativamente) pristino, buona parte è inabitabile, perché situata nelle zone artiche, desertiche o di alta montagna (LeB. Hooke, 2012).

Tre aspetti devono essere considerati con attenzione. Il primo riguarda l'intrusione nelle grandi foreste, e in particolari di quelle pluviali, la cui integrità è una garanzia dell'equilibrio bio-naturale. Il secondo consiste nell'intensificazione del popolamento negli habitat più precari, in particolare nelle aree costiere, fluviali o lacuali. Il terzo aspetto attiene all'esplosione dei processi di urbanizzazione.

Per quanto riguarda la deforestazione, questa ha colpito i maggiori bacini fluviali in America, Africa ed Asia. Nella Amazonia "classica" (3.6 $\mathrm{km}^{2}$ ), la superficie deforestata è cresciuta dal due per cento nel 1980, al 12 per cento nel 2010, mentre in questo periodo la popolazione è crescita di due volte e mezzo (da 5.9 a 14.8 milioni) per l'alta immigrazione. La deforestazione per ottenere spazi residenziali, per le infrastrutture, le coltivazioni, i pascoli, i campi minerari è strettamente legata all'immigrazione.

Le foreste pluviali hanno un ruolo cruciale nella conservazione dell'equilibrio ambientale; nel moderare le emissioni di gas serra, nel mantenere l'integrità delle riserve d'acqua; nel proteggere la biodiversità. Il fondamentale problema per la protezione del manto forestale è il fatto che gli alberi hanno più valore se vengono tagliati anziché lasciati vivere, e che i pascoli e le colture hanno più valore delle foreste vergini. Sono perciò necessarie norme stringenti. 
La densità demografica e la crescita della popolazione sono più alte nelle aree costiere che non in quelle interne; le prime hanno più valore e sono più attrattive e funzionali (storicamente, le grandi città si sono sviluppate lungo le coste o sulle rive delle maggiori vie d'acqua). Le zone costiere favoriscono le attività commerciali e quelle industriali, e attraggono flussi di immigrazione; necessitano di infrastrutture; stimolano l'urbanizzazione. Ma molte aree - specialmente quelle costiere con bassa elevazione sul mare- oltre ad essere attrattive e pregiate, sono anche fragili e vulnerabili (si pensi alle 200 mille e più vittime provocate dallo tsunami del 2004 nel Sud-est asiatico), e questa condizione si aggraverà con il rialzo del livello del mare per il riscaldamento globale.

Una recente pubblicazione delle Nazioni Unite afferma "delle 1692 città con almeno 300 mille abitanti (2014), 944 (56 per cento) sono ad alto rischio per l'esposizione ad almeno uno di sei tipi di disastri naturali (cicloni, inondazioni, siccità, terremoti, frane e eruzioni vulcaniche), come si desume dall'esperienza dei disastri naturali avvenuti nell'ultima parte del XX secolo. Nell'insieme, le città con alta esposizione ai disastri naturali ospitavano 1.4 miliardi di persone nel 2014 (United Nations, 2016). Nel 2050 c'erano due "mega-città" di oltre 10 milioni di abitanti nel mondo, ma nel 2016 ce ne sono 31, delle quali 24 nei paesi in via di sviluppo. Il moderno processo di "mega-urbanizzazione" è stato compresso in un breve periodo, in maniera disordinata e spesso anarchica. Le conseguenze ambientali riguardano soprattutto l'inquinamento atmosferico — con le relative conseguenze negative per la salute - e quello delle acque, con effetti che si propagano nell'ecosistema ben aldilà del territorio delle mega-città. Si aggiunga poi lo spreco ed il degrado dello spazio. E poiché la crescita delle grandi conurbazioni avviene più velocemente di quella delle aree urbane nel loro complesso, gli effetti negativi sull'ecosistema sono destinati ad aggravarsi ulteriormente, a meno che non si intervenga con robuste misure correttive.

\section{Ancora Malthus?}

Sì, Malthus ancora. Il mondo ha dimensioni finite: può darsi che non ci siamo limiti per la produzione delle sussistenze, come pensava Malthus. Ma terra, spazio, aria e acqua sono limitati, e sono risorse che vanno bene amministrate. E ciò significa robuste azioni di governo, particolarmente a livello internazionale. E il mondo potrebbe essere in migliori condizioni, verso il 2100, con una popolazione di 10 anziché di 11 miliardi. 


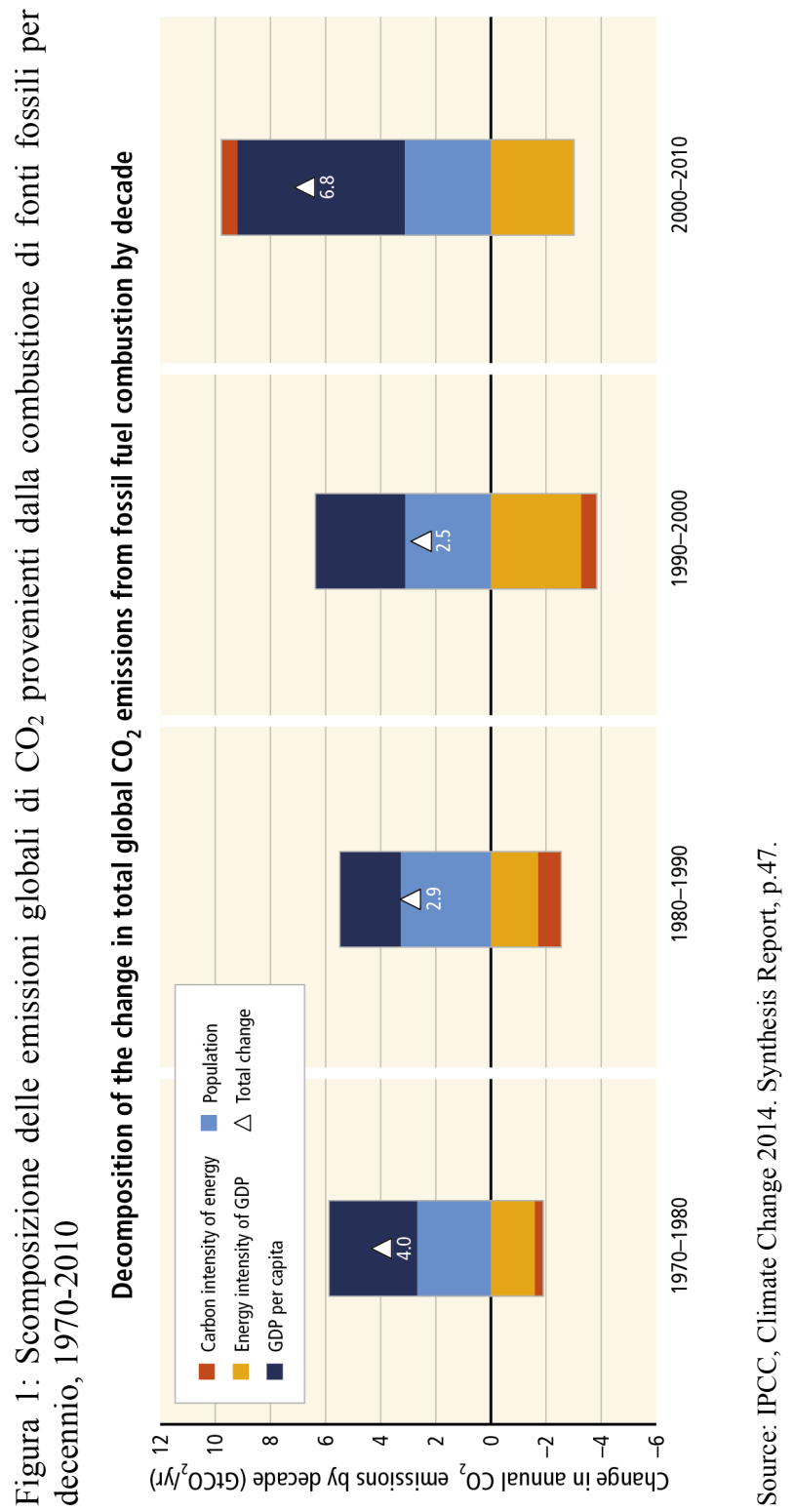




\section{RIFERIMENTI BIBLIOGRAFICI}

Bautista Alberdi, Juan, 1852, Bases y puntos de partida para la organización politica de la República de Argentina, disponible en http://www.hacer.org/pdf/Bases. pdf, p.5.

Ehrlich, Paul, 1968, The Population Bomb, Ballantine Books, New York, Prologue.

Grossman, Richard, 2017, The world in which the next 4 billion people will live, N-IUSSP, November 13, 2017.

International Panel on Climate Change, 2014, Climate Change 2014. Synthesis Report, p.47, (IPCC), disponible en http://www.ipcc.ch/report/ar5/syr/

Lam, David, 2017, The world's next 4 billion people will differ from the previous 4 billion, N-IUSSP, July 24.

LeB. Hooke, R. Martin-Duque, J.E. and Pedraza, J., 2012, Land transformation by humans: a review, GSA Today, vol 22, issue12, 2012.

Livi Bacci, Massimo, 2015a, Il pianeta stretto, Il Mulino,Bologna.

Livi Bacci, Massimo, 2015b, Pauperia and Tycoonia: population and sustainability, N-IUSSP, July 14.

Livi Bacci, Massimo, 2017, Our Shrinking Planet, Polity Press, Cambridge.

Malthus, Thomas R., 1970, An Essay on the principle of population,Penguin Books, Harmondsworth, 1970 , p. 71.

Martine, George, s./f., Global population, development aspirations and fallacies, N-IUSSP.

Meadows, D.H, Meadows, D.L., Randers, J., Behrens III, W.W., 1972, The Limits to Growth, Universe Books, New York.

Ratzel, Friedrich, 1897, Politische Geographie.

Schumpeter, Joseph A., 1959, Storia dell'analisi economica, vol. I, Einaudi, Torino, pp. 303-304

Spengler, J., 1938, France faces depopulation, p. 123, n. 1. Duke University Press, Durham.

United Nations, 2016, The World's Cities in 2016 - Data Booklet, http://www. un.org/en/development/desa/population/publications/pdf/urbanization/the worlds_cities_in_2016_data_booklet.pdf

United Nations, 2017, World Population Prospects. The 2017 Revision, New York. 


\section{SOMMARIO CURRICULUM DELL'AUTORE}

\section{Massimo Livi Bacci}

Es profesor de Demografía en la Universidad de Florencia y ha formado demógrafos en varios países americanos entre los que destacan Estados Unidos, México, Brasil y también en algunos países europeos. Su labor científica se ha centrado en diversos aspectos de la Demografía histórica y la Demografía actual. Es partidario de un enfoque interdisciplinario de los estudios de población y considera que la expansión a otros campos, más allá de la Demografía, pero desde ella, es esencial para obtener una explicación correcta de los grandes temas. Mantiene dos líneas de investigación: Poblaciones nativas de Iberoamérica después de la conquista, investigación en el ámbito de la Demografía histórica e Interacción entre los cambios demográficos y las políticas sociales. Ha publicado numerosos libros, ensayos y artículos y ha dirigido grupos de investigación. Es cofundador de sociedades científicas, en colaboración con instituciones públicas y privadas, tanto en Italia como en el resto del mundo.

Dirección electrónica: livi@disia.unifi.it

Artículo recibido el 11 de enero de 2018 y aprobado el 19 de febrero de 2018. 